\title{
Zum Update des Meikirch-Modells: eine Schützenhilfe vom Übervater
}

\author{
Jann Schwarzenbach \\ Dr. med., Facharzt für Allgemeinmedizin, Mitglied FMH
}

Im Gegensatz zur kurzlebigen Medizin ist es in den Geisteswissenschaften eher normal, seine eigenen Gedanken mit denjenigen richtungsweisender früherer Autoren in Verbindung zu bringen. In diesem Sinne versteht sich mein Versuch sozusagen als flankierende Massnahme -, das aktuelle Update zum Meikirch-Modell (MM) philosophisch gewürzt, ein wenig schmackhafter zu machen. Dabei von einem geistigen Übervater wie Platon Schützenhilfe anzunehmen, darf nicht als Vermessenheit gelten, im Gegenteil: Es ist Ausdruck der eigenen Bescheidenheit ein Antidot gegen jede überhebliche Illusion, selbst das sprichwörtliche Pulver erfunden zu haben.

\section{Das Gesundheitswesen: ein chronischer Patient}

Unser Gesundheitswesen wird dem Schlagwort «nomen est omen" nicht mehr gerecht. Hin- und hergerissen zwischen Einzelinteressen und allgemeiner Tragbarkeit, ethischem Anspruch und Machbarkeitswahn, ist es, in sich uneins, selbst zum Patienten geworden. Man spürt, dass sein traditionelles Anliegen, die Gesundheit, zu Gunsten einer ökonomisch-technischen Fortschrittslogik, zunehmend aus dem Fokus geraten ist, und so suchen manche seit geraumer Zeit nach dem berühmten "Reset-Knopf» zur Neuorientierung. Salben und Pflastern, da und dort, bei Planung und Tarifen, brachte bisher kaum Linderung. Ein radikales Umdenken drängt sich auf. Das MM bietet sich an.

Man spürt, dass die Gesundheit, zu Gunsten einer ökonomisch-technischen Fortschrittslogik, zunehmend aus dem Fokus geraten ist.

\section{Gesundheit als Kultur: ein Bildungsauftrag}

Ein Paradigmenwechsel braucht neue Formen der Kommunikation, er spricht sozusagen seine eigene Sprache, und es gibt ihn daher auch nicht sofort auf Rezept, sondern seine Zulassung erfordert erst einmal die Schaffung klarer und wiederholt anwendbarer Be- griffe. So beschäftigt sich denn auch das MM primär mit dem Versuch, die Gesundheit auf eine ganz neue Art $\mathrm{zu}$ definieren. Sie wird da als ein dynamisches Gleichgewicht verstanden, wo die patienteneigenen, biologisch gegebenen, und die erworbenen Potentiale stets neu mit den Anforderungen des Lebens in Einklang gebracht werden müssen. Die Krankheiten können demnach als in diesem Orchester auftretende Dissonanzen aufgefasst werden, und ihre Behandlung soll sich mittels breit gefasster Massnahmen darauf konzentrieren, das ganze System wieder ins Lot zu bringen. Um genügende Wirkung zu erreichen, müsste das MM von möglichst allen mit dem Gesundheitssystem verbundenen Personen und Institutionen angewendet werden, und idealerweise auch in die nichtmedizinischen Lebensbereiche Einzug halten. Dazu braucht es eine Einbindung ins allgemeine Bildungswesen. Es geht hier nämlich um nicht weniger als um die Vermittlung einer neuen Gesundheitskultur. Zu diesem Zweck müssen dann auch ergänzende Stimmen zu derjenigen der medizinischen Wissenschaft gehört werden: Die der Politik und des Gesetzgebers und - warum nicht - auch jene der Philosophie.

\section{Gesundheit als System: von der Idee in die Praxis}

Insgesamt hat das MM durchaus Systemcharakter, und erhebt daher auch Anspruch auf allgemeine Gültigkeit 
und breite praktische Anwendbarkeit. Es ist damit verwandt mit verschiedenen philosophischen Konstrukten, die oftmals von einem modellhaften Grundgedanken ausgehen, der sich dann in verschiedensten Gebieten wiederfinden lässt. So steht beispielsweise im Denken Platons (ca. 428-348 v.Chr.) auch ein Wert im Mittelpunkt des Interesses, statt der Gesundheit ist es hier der Gesamtzustand eines geglückten Lebens, die Eudaimonie. Nach der Ansicht des Philosophen wirken in unserem Innenleben zwei verschiedene Schubkräfte: Der Drang zu tatkräftiger Verwirklichung und individueller Bedeutung, und derjenige zur Befriedigung physischer Bedürfnisse und Begehrlichkeiten. Diese ungezähmten Energien massvoll zu nutzen ist die Aufgabe einer dritten Kraft, die der tugendhaften

\section{Platon will sein Modell der menschlichen Seele - den Promotoren des MM nicht unähnlich - in der Gesellschaft praktisch verwirklicht wissen.}

Vernunft, die das dynamische Seelenleben kompensatorisch zügelt und als gelingendes Ganzes in innerer Harmonie zusammenzuhalten weiss. Der Mensch ist dann nicht in der Eudaimonie, wenn ein Ungleichgewicht besteht zwischen seinem Anspruch an ein gelingendes Leben und den eigenen innerseelischen energetischen Potentialen, respektive wenn Letztere nur mangelhaft durch die Vernunft moduliert werden. Die Parallelen zum MM drängen sich da geradezu auf: es genügt, die obgenannten Schubkräfte mit dem biologisch gegebenen und die einende Weisheit mit dem persönlich erworbenen Potential zu ersetzen, die in enger Zusammenarbeit den Anforderungen des Lebens gerecht werden müssen. Platon versteht sein Modell der menschlichen Seele aber nicht nur als theoretische "Trouvaille», sondern will es - den Promotoren des MM nicht unähnlich - in der Gesellschaft praktisch verwirklicht wissen. Seine daraus resultierende elitäre und autokratische Utopie eines idealen Staates wurde von anderen Denkern allerdings scharf kritisiert. Er postuliert nämlich eine hierarchische Standesordnung als Spiegelbild der drei obgenannten innerseelischen Prinzipien. Für die physiologischen Bedürfnisse sorgen Bauern, Handwerker und Kaufleute, während Wächter und Krieger zur Verteidigung der gesellschaftlichen Macht- und Sicherheitsansprüche ihren Einsatz finden. Zur Regierungsgewalt gelangen einzig diejenigen, die durch philosophische Schulung die dazu benötige Weisheit erreicht haben. Nur so seien sie nämlich in der Lage, ausgleichend $\mathrm{zu}$ wirken, und die anderen Stände zu Tugend und Zufriedenheit, eben zur individuellen Eudaimonie, anzuleiten und so die ganze Gesellschaft im Zeichen der Gerechtigkeit, der höchsten Tugend eines Staates, zusammenzuhalten.

\section{Das Ei des Kolumbus?}

Obwohl die vom MM eingeforderten gesellschaftlichen Anpassungen ganz anderer Natur sind als diejenigen von Platos Staatsmodell, zeigt doch gerade dieses Letztere, dass idealisierende Denksysteme in ihrer praktischen Anwendung nicht frei von Nebenwirkungen sind. Sie müssen daher einer ständigen konstruktiven Kritik offen bleiben. Das gilt auch für das MM und dessen nicht unbescheidenen Anspruch auf einen umfassenden Paradigmenwechsel. Wie bereits erwähnt, benötigt ein radikales Umdenken auch eine geeignete Kommunikation und eine angemessene Zeit, um alle «Endkapillaren» der Gesellschaft $\mathrm{zu}$ erreichen. Die Diffusion ist eben ein langsamer, durch mehrere Kanäle erfolgender Prozess. In diesem Sinne könnte denn, zusätzlich zum aktuellen Update, auch der philosophische Ansatz dem MM behilflich sein, vielleicht doch noch zum Ei des Kolumbus zu werden. 